\title{
Construction of S-doped hierarchical porous carbons by acid- free treatment strategy for supercapacitors
}

\author{
Yang Lei ${ }^{1}$, Jin BiYu ${ }^{1}$, Li HongQiang ${ }^{1}$, Wu TingTing ${ }^{1}$, He XiaoJun ${ }^{1 *}$ \\ ${ }^{1}$ School of Chemistry and Chemical Engineering, Anhui University of Technology, Maanshan, Anhui, China
}

\begin{abstract}
The pollution from acid treatment process in the preparation process of hierarchical porous carbons is a substantial challenge for industrial application of supercapacitors (SCs), which necessitates the development of green alternative technologies. In this work, S-doped hierarchical porous carbons (S-HPCs) are prepared from cheap coal tar pitch by a less harmful in situ $\mathrm{KHCO}_{3}$ activation strategy. The sample obtained at $800^{\circ} \mathrm{C}$ (S-HPC 800$)$ possesses 3D framework structure with hierarchical pores, large specific surface area $\left(1485 \mathrm{~m}^{2} \mathrm{~g}^{-1}\right)$ and $\mathrm{O}$, S-containing functional groups. Due to these synergistic characteristics, S$\mathrm{HPC}_{800}$ as supercapacitor electrode exhibits high specific capacitance of $246 \mathrm{~F} \mathrm{~g}^{-1}$ at $0.1 \mathrm{~A} \mathrm{~g} \mathrm{~g}^{-1}$ with a capacitance retention of $68.3 \%$ at $40 \mathrm{~A} \mathrm{~g}^{-1}$ and excellent cycle stability with $96.7 \%$ capacitance retention after 10,000 charge-discharge cycles. This work provides an environmentally friendly approach to prepare advanced carbon-based electrode materials from industrial by-products for energy storage devices.
\end{abstract}

\section{Introduction}

Owing to the exhaustion of fossil fuels and the worsening environmental pollution, stable and renewable energy sources are highly desired in recent years [1]. Therefore, electrochemical energy storage (EES) devices which can efficiently utilize clean resources attract the researchers' attention. Supercapacitors (SCs) have been widely studied as a typical EES device due to their rapid charge-discharge rate, high power density and long cycle life [2-4]. According to the energy storage mechanism, SCs can be divided into electric double layer capacitors (EDLCs) and pseudocapacitors [5]. EDLCs and pseudocapacitors store energy through reversible ion adsorption and fast redox reactions at the surface of electrode materials, respectively. Therefore, electrode materials play a vital role in the performance of SCs. Carbon materials are considered as the most promising electrode materials in virtue of their good electrical conductivity and high specific surface area [6]. However, pure carbon electrode materials still have great limitations in terms of electrochemical performance. Structural optimization and surface modification of pure carbon materials suit the remedy to the case.

In order to achieve high performance carbon electrode materials, an effective method is to design hierarchical porous carbons (HPCs) with micro-, meso- and macropores. Micropores can provide a large number of active sites for reversible ion adsorption [7]. Mesopores act as electrolyte container which is conducive to the spread and transmission of ions [8]. Macropores can be used as ion-buffering reservoirs which are benefit to mass transport of ions [9]. In addition, heteroatom doping (such as $\mathrm{N}, \mathrm{P}, \mathrm{S}$ and $\mathrm{B}$ ) which can improve wettability and increase pseudocapacitance of HPCs is another efficient method to enhance electrochemical performance of electrode materials [10-12]. Generally, HPCs can be prepared by using hard template such as $\mathrm{SiO}_{2}$, nano- $\mathrm{CuO}$, nano- $\mathrm{ZnO}$ and nano- $\mathrm{Fe}_{2} \mathrm{O}_{3}$ [13]. However, the using of corrosive $\mathrm{HF}$ or volatile $\mathrm{HCl}$ is inevitable to remove hard templates, which results in environmental pollution. Therefore, the exploitation of a more environmentally friendly method is still a 'must-do' agenda for pushing commercialization of SCs.

Coal tar pitch (CTP) is a residue fraction from the distillation of coal tar, which possesses various kinds of polycyclic aromatic hydrocarbons with viscous and thermoplastic characteristics [14]. In this work, we report an eco-friendly method to prepare S-doped hierarchical porous carbons (S-HPCs) from CTP. The sample obtained at $800^{\circ} \mathrm{C}\left(\mathrm{S}-\mathrm{HPC}_{800}\right)$ featuring three-dimensional (3D) structure is composed of interconnected carbon capsules and sheets with abundant active sites for fast ion adsorption. Moreover, S-doped can further improve the electrical conductivity and boost the surface wettability of $\mathrm{S}-\mathrm{HPC}_{800}$. Benefiting from such constructive characteristics, $\mathrm{S}-\mathrm{HPC}_{800}$ as the electrode material for SC exhibits high specific capacitance, good rate capability and superior cycle stability.

\section{Materials and Methods}

\subsection{Preparation of samples}

CTP was obtained from Maanshan Iron \& Steel Co. Ltd. (China), and other chemicals were received from Aladdin Co. In a typical case, $2 \mathrm{~g} \mathrm{CTP}, 12 \mathrm{~g} \mathrm{KHCO}_{3}$ and $2.5 \mathrm{~g}$

*Corresponding author: xjhe@ahut.edu.cn 
$\mathrm{Na}_{2} \mathrm{SO}_{4}$ were mixed with continuously stirring for $30 \mathrm{~min}$. Then, the resulting mixture was heated in a horizontal

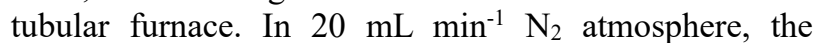
mixture was firstly heated to $150^{\circ} \mathrm{C}$ at $5^{\circ} \mathrm{C} \mathrm{min}{ }^{-1}$ for 30 min, followed by being heated to $\mathrm{X}^{\circ} \mathrm{C}$ (X stands for 750 , 800 and 850 ) at $5^{\circ} \mathrm{C} \mathrm{min}^{-1}$ for $1 \mathrm{~h}$. After cooling down naturally, the as-prepared samples were purified merely by distilled water washing and dried at $100^{\circ} \mathrm{C}$ overnight. The final products were denoted as $\mathrm{S}-\mathrm{HPC}_{\mathrm{X}}$. $\mathrm{HPC}_{800}$ without adding $\mathrm{Na}_{2} \mathrm{SO}_{4}$ was prepared by the similar procedure as control.

\subsection{Characterization}

Field emission scanning electron microscopy (FESEM, NanoSEM430, USA) and transmission electron microscopy (TEM, JEOL-2100, Japan) were employed to investigate the morphology of HPCs. Energy dispersive spectroscopy (EDS) was used to test surface elements. Nitrogen adsorption-desorption isotherms were conducted at $-196^{\circ} \mathrm{C}$ on an Autosorb-IQ system (Quantachrome, USA). The specific surface area $\left(\mathrm{S}_{\mathrm{BET}}\right)$ was calculated by the Brunauer-Emmett-Teller (BET) method. The pore diameter was analysed from the adsorption branches of the isotherms using the density functional theory (DFT) method. Raman spectroscopy (RamHR800) was used to examine the defect degree of the samples. X-ray photoelectron spectroscopy (XPS, ThermoESCALAB250) was used to measure the contents of elements and functionalities.

\subsection{Electrochemical measurement}

Firstly, HPCs $(90 \mathrm{wt} \%)$ and polytetrafluoroethylene $(10 \mathrm{wt} \%)$ were mixed together in deionized water. Secondly, the mixture was rolled and then made into circular films with diameter of $12 \mathrm{~mm}$. Thirdly, the asprepared films were dried at $110{ }^{\circ} \mathrm{C}$ for $2 \mathrm{~h}$ in vacuum oven, followed by being pressed onto nickel foams to obtain the electrodes. Before being assembled in supercapacitors, the electrodes were soaked in $6 \mathrm{M} \mathrm{KOH}$ electrolyte for $2 \mathrm{~h}$ under vacuum. Finally, the immersed electrodes were assembled into a symmetrical button supercapacitor.

The cyclic voltammetry (CV) test was carried out on a CHI760E electrochemical workstation (Shanghai, China) and the galvanostatic charge-discharge (GCD) measurement was performed on a supercapacitance test system (SCTs, Arbin Instruments, USA). The electrochemical impedance spectroscopy (EIS) test was carried out on a Solartron impedance analyzer (Solartron Analytical, SI1260, UK). The specific capacitance ( $C, \mathrm{~F} \mathrm{~g}^{-}$ ${ }^{1}$ ) of HPC electrodes was calculated from the GCD curves by the following Equation (1).

$$
C=4 I \Delta t /(m \Delta V)
$$

Where $I(\mathrm{~A}), \Delta V(\mathrm{~V}), \Delta t(\mathrm{~s})$ and $m(\mathrm{~g})$ signifies the discharge current, the discharge voltage after the IR drop, the discharge time and total mass of active materials, respectively. The energy density $\left(E, \mathrm{Wh} \mathrm{kg}^{-1}\right)$ and average power density $\left(P, \mathrm{~W} \mathrm{~kg}^{-1}\right)$ of HPC electrodes were calculated via Equation (2) and Equation (3), respectively.

$$
\begin{gathered}
E=C V^{2} / 28.8 \\
P=E / \Delta t
\end{gathered}
$$

Where $V(\mathrm{~V}), \Delta t(\mathrm{~h})$ stands for the discharge voltage after the IR drop and the discharge time, respectively.

\section{Results \& Discussion}

The nitrogen adsorption-desorption isotherms of four HPC samples are shown in Fig. 1a. All the nitrogen adsorption-desorption isotherms of the HPCs exhibit strong adsorption at $P / P_{0}<0.01$, small hysteresis loops at $0.4<P / P_{0}<0.9$ and little tail at $P / P_{0}>0.9$, which suggests the existence of a large number of micropores, a moderate number of mesopores and a small number of macropores [15]. It is well known that macropores, mesopores and micropores avail for ion-buffering, ion transport and ion adsorption, respectively [16]. Fig. 1b indicates that the pore size of HPCs is mainly concentrated in $0.5 \sim 4 \mathrm{~nm}$. With the increasing of temperature, the $\mathrm{S}_{\mathrm{BET}}$ of S-HPCs first raised from $1199 \mathrm{~m}^{2} \mathrm{~g}^{-1}$ to $1485 \mathrm{~m}^{2} \mathrm{~g}^{-1}$, and then fell to $1257 \mathrm{~m}^{2} \mathrm{~g}^{-1}$, while the average pore diameter $\left(\mathrm{D}_{\mathrm{ap}}\right)$ increased from $2.53 \mathrm{~nm}$ to $2.86 \mathrm{~nm}$ (Table 1). The reason for such changes of $S_{\mathrm{BET}}$ and $D_{a p}$ is that the strong activation of $\mathrm{KHCO}_{3}$ lead to the collapse of some micropores and mesopores under high temperature conditions. In addition, the $\mathrm{S}_{\mathrm{BET}}$ of S-HPC $\mathrm{C}_{800}\left(1485 \mathrm{~m}^{2} \mathrm{~g}^{-}\right.$ ${ }^{1}$ ) is much higher than $\mathrm{HPC}_{800}\left(998 \mathrm{~m}^{2} \mathrm{~g}^{-1}\right)$ (Table 1), which demonstrates that $\mathrm{Na}_{2} \mathrm{SO}_{4}$ can be used as an auxiliary activator and the doping of $\mathrm{S}$ is in favour of the formation of edge defects. The foregoing results suggest that the pore structure parameters of HPCs can be tuned by changing the activation temperature and introducing $\mathrm{S}$ atom.
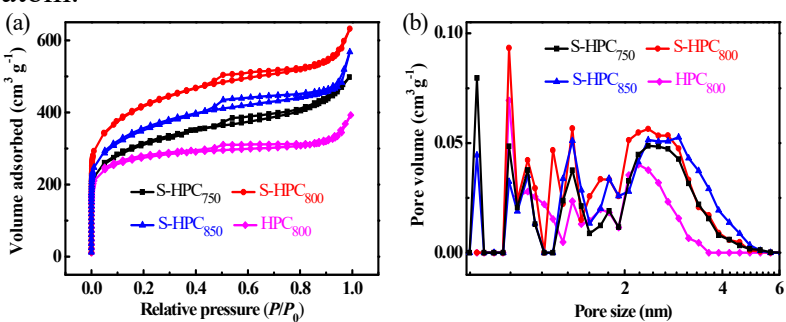

Fig1. (a) Nitrogen adsorption-desorption isotherms; (b) pore size distribution of HPCs.

Table1. Pore structure parameters of HPCs.

\begin{tabular}{ccccc}
\hline Samples & $\begin{array}{c}\mathrm{D}_{\mathrm{ap}} \\
(\mathrm{nm})\end{array}$ & $\begin{array}{c}\mathrm{S}_{\mathrm{BET}} \\
\left(\mathrm{m}^{2} \mathrm{~g}^{-1}\right)\end{array}$ & $\begin{array}{c}\mathrm{V}_{\mathrm{t}} \\
\left(\mathrm{cm}^{3} \mathrm{~g}^{-1}\right)\end{array}$ & $\begin{array}{c}\mathrm{V}_{\text {mic }} \\
\left(\mathrm{cm}^{3} \mathrm{~g}^{-1}\right)\end{array}$ \\
\hline $\begin{array}{c}\text { S- } \\
\mathrm{HPC}_{750}\end{array}$ & 2.53 & 1199 & 0.76 & 0.33 \\
$\begin{array}{c}\mathrm{S}- \\
\mathrm{HPC}\end{array}$ & 2.64 & 1485 & 0.98 & 0.45 \\
$\quad \mathrm{~S}-$ & & & & 0.35 \\
$\begin{array}{c}\text { HPC } \\
\text { HPC }\end{array}$ & 2.86 & 1257 & 0.90 & 0.29 \\
\hline
\end{tabular}

The FESEM images in Fig. 2a-e show that the 3D framework structure of $\mathrm{HPC}_{800}$ and $\mathrm{S}_{-} \mathrm{HPC}_{800}$ are consisted of interconnected carbon sheets and capsules with opened pores. The EDS mapping images of $\mathrm{HPC}_{800}$ (Fig. 2f) display a relatively homogeneous distribution of $\mathrm{C}, \mathrm{O}$ and $\mathrm{N}$ elements. The reason for the existence of $\mathrm{N}$ element is that there is a small amount of N-containing 
substance in CTP. The TEM images further prove that there are interconnected carbon sheets and capsules with large pores in $\mathrm{S}_{-} \mathrm{HPC}_{800}$ structure (Fig. 3a, b, d, e). Besides, the HRTEM images show that S-HPC 800 possesses local graphitization stripes. The EDS mapping images of S$\mathrm{HPC}_{800}$ display a relatively homogeneous distribution of $\mathrm{C}, \mathrm{O}, \mathrm{N}$ and $\mathrm{S}$ elements (Fig. 1g), which confirms that $\mathrm{S}$ atom was successfully doped in S-HPC 800 .

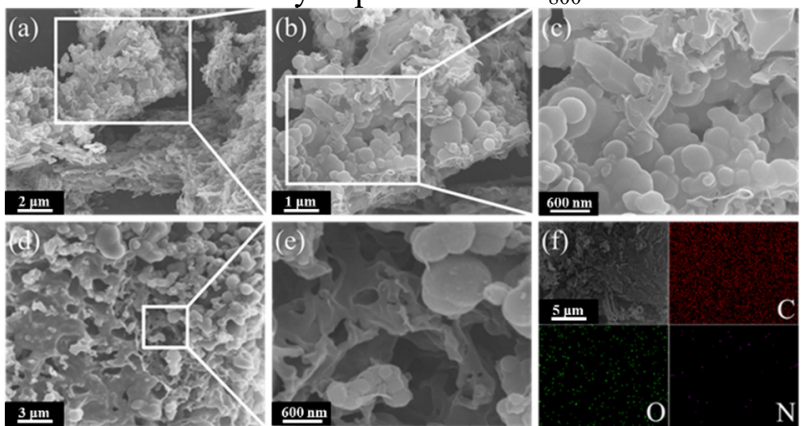

Fig2. FESEM images of (a-c) $\mathrm{HPC}_{800}$ and (d, e) S-HPC 800 ; (f) EDS mapping images of $\mathrm{HPC}_{800}$.

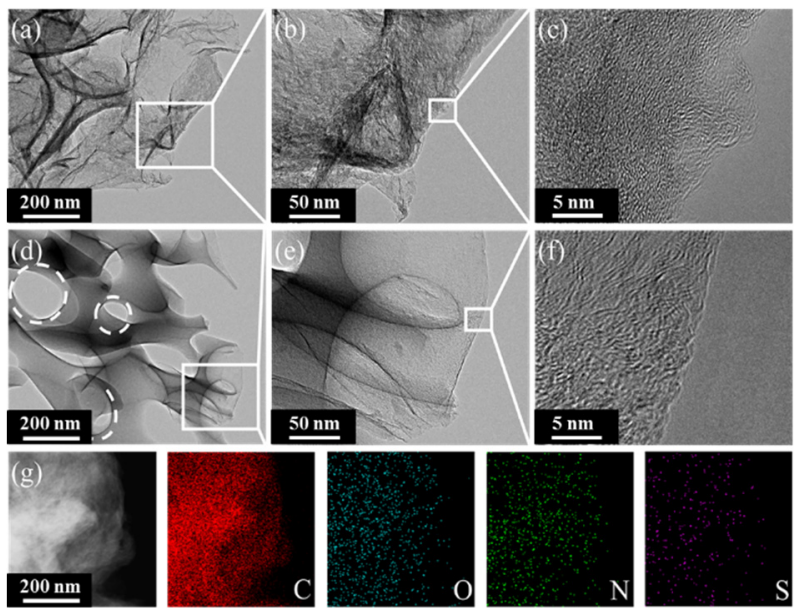

Fig3. TEM images of (a-f) S-HPC 800 ; (g) EDS mapping images of S-HPC 800 .

The Raman spectra of HPCs display two strong characteristic peaks at ca. $1343 \mathrm{~cm}^{-1}$ and ca. $1587 \mathrm{~cm}^{-1}$, corresponding to D-band and G-band, respectively (Fig. 4a). The former is related to the defects and disorder structures, while the latter can be assigned to the graphitic structures [17]. The peak intensity ratio of the D-band to G-band $\left(I_{\mathrm{D}} / I_{\mathrm{G}}\right)$ of S-HPC 800 (1.01) is higher than that of S$\mathrm{HPC}_{750}$ (0.95), S-HPC 850 (0.97) and $\mathrm{HPC}_{800}$ (0.93), which confirms that $\mathrm{S}-\mathrm{HPC}_{800}$ possesses the most defects among the four HPCs. The full XPS survey scan spectrum of S$\mathrm{HPC}_{800}$ suggests the presence of $\mathrm{C}(284.6 \mathrm{eV}), \mathrm{O}(532.1$ eV), N (400.0 eV), and S (164.4 eV) (Fig. 4b). The highresolution $\mathrm{O} 1 \mathrm{~s}$ spectrum of $\mathrm{S}-\mathrm{HPC}_{800}$ witnesses three peaks: $\mathrm{O}-\mathrm{H}(535.5 \mathrm{eV}), \mathrm{C}-\mathrm{O}(532.5 \mathrm{eV})$ and $\mathrm{C}=\mathrm{O}(531.2$ eV) (Fig. 4c) [18]. In addition, the high-resolution $\mathrm{S} 2 \mathrm{p}$ spectrum of S-HPC 800 was fitted into three peaks with the binding energies of $163.2 \mathrm{eV}, 164.9 \mathrm{eV}$ and $168.6 \mathrm{eV}$, corresponding to $\mathrm{S} 2 \mathrm{p}_{3 / 2}, \mathrm{~S} 2 \mathrm{p}_{1 / 2}$ and $\mathrm{SO}_{\mathrm{X}}$, respectively (Fig. 4d) [19]. The doping of these heteroatoms can not only improve the wettability of S-HPC ${ }_{800}$ but also provide additional pseudocapacitance, as a result, remarkably improve capacity performance.
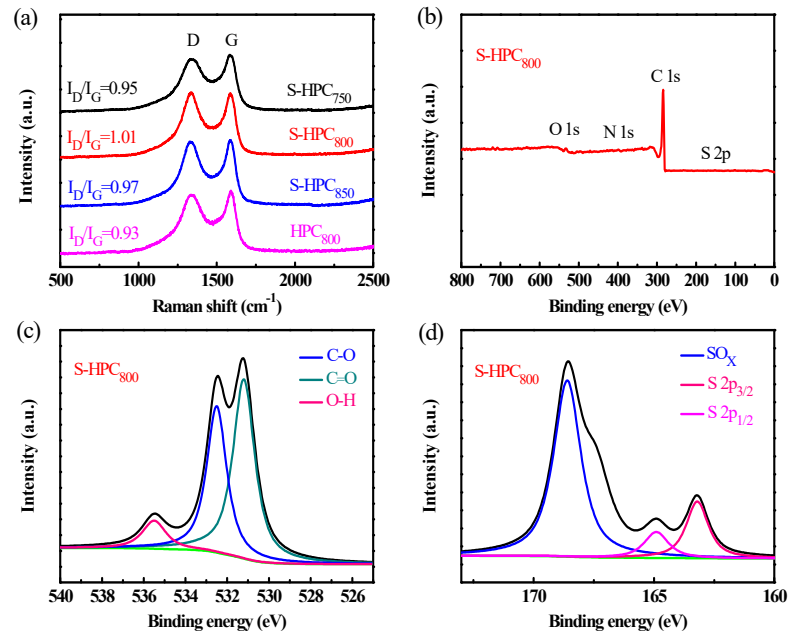

Fig4. (a) Raman spectra of HPCs; (b) full XPS, (c) O 1s and (d) S 2p spectra of S-HPC 800 .
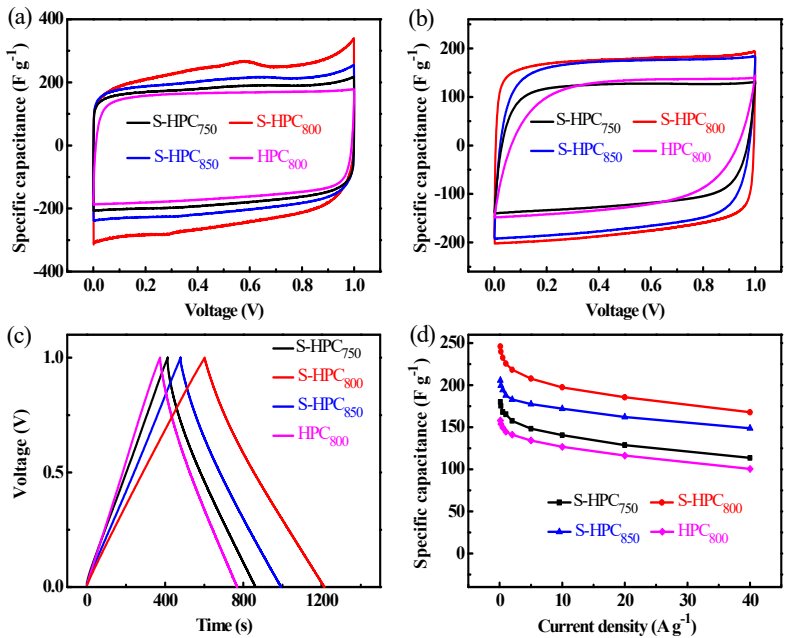

Fig5. CV curves of HPC electrodes at (a) $5 \mathrm{mV} \mathrm{s}^{-1}$ and (b) 200 $\mathrm{mV} \mathrm{s}^{-1}$; (c) GCD curves of HPC electrodes at $0.1 \mathrm{~A} \mathrm{~g}^{-1}$; (d) specific capacitance at different current densities.

The electrochemical performance of HPC electrodes were evaluated in symmetric coin-type SCs using $6 \mathrm{M}$ $\mathrm{KOH}$ aqueous solution as electrolyte. The CV curves of HPC electrodes show a rectangular shape at a scan rate of $5 \mathrm{mV} \mathrm{s}^{-1}$. Fortunately, no obvious distortions are observed when the scan rate increases to $200 \mathrm{mV} \mathrm{s}^{-1}$ (Fig. 5a, b), displaying the ideal EDLC behaviour and good rate performance of HPC electrodes [20]. At the current density of $0.1 \mathrm{~A} \mathrm{~g}^{-1}$, all the GCD curves of HPC electrodes show symmetrical triangles (Fig. 5c), which indicates ideal EDLC behaviour [21]. Fig. 5d shows that the specific capacitance of $\mathrm{S}-\mathrm{HPC}_{800}$ electrode is always higher than that of other three HPC electrodes at the same current density. The specific capacitance of S-HPC 800 is $246 \mathrm{~F} \mathrm{~g}^{-1}$ at $0.1 \mathrm{~A} \mathrm{~g}^{-1}$ with a capacitance retention of $68.3 \%$ at $40 \mathrm{~A}$ $\mathrm{g}^{-1}$. The hierarchical porous structure and synergistic effect of $\mathrm{S}$ doping are of great significance for the improving specific capacitance and rate performance of S$\mathrm{HPC}_{800}$.

The energy densities of S-HPC ${ }_{800}$-based SC are 8.5 and $3.3 \mathrm{Wh} \mathrm{kg}^{-1}$ at the power density of 51.6 and 11520.7 $\mathrm{W} \mathrm{kg}^{-1}$, respectively (Fig. 6a), which confirms its potential application. In addition, S-HPC 800 -based $\mathrm{SC}$ exhibits a long-term cycle stability, whose capacitance retention 
stabilized at $96.7 \%$ even after 10,000 cycles at $5 \mathrm{~A} \mathrm{~g}^{-1}$ (Fig. 6b). Nyquist plots of HPC electrodes in $6 \mathrm{M} \mathrm{KOH}$ electrolyte are shown in Fig. 6c. It can be found that $\mathrm{S}$ $\mathrm{HPC}_{800}$ electrode presents a straight line paralleling to the $\mathrm{Y}$ axis at low-frequency part, manifesting the ideal EDLC behaviour [22]. At high frequency region, the small Xintercept and semicircle demonstrate that $\mathrm{S}-\mathrm{HPC}_{800}$ electrode has very low intrinsic ohmic resistance $\left(R_{\mathrm{S}}\right)$ and charge transfer resistance $\left(R_{\mathrm{ct}}\right)$, which further gives rise to the rapid transmission and diffusion of electrolyte ions.
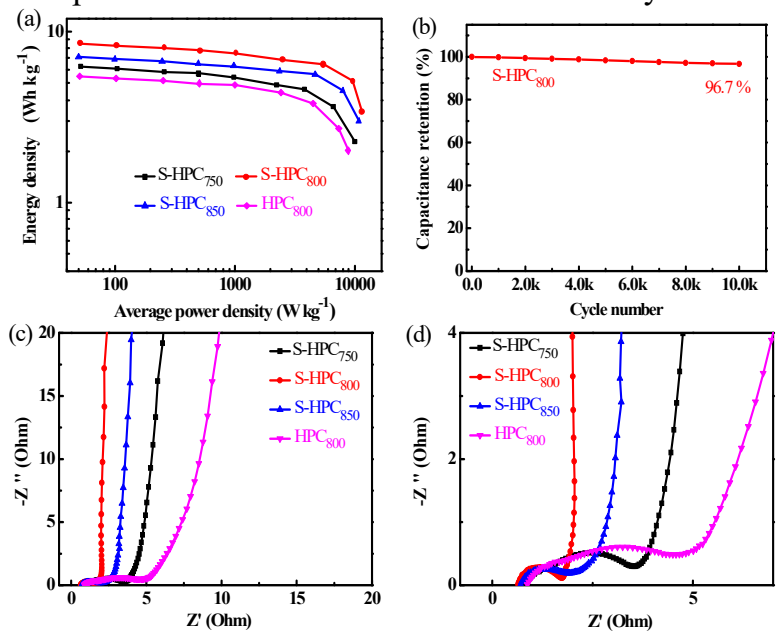

Fig6. (a) Ragone plots of HPC-based SCs; (b) capacitance retention of S-HPC 800 -based SC after 10,000 cycles at $5 \mathrm{~A}$ $\mathrm{g}^{-1}$; (c) Nyquist plots of HPC electrodes and (d) corresponding Nyquist plots of high frequency region.

\section{Conclusions}

In summary, simple and eco-friendly in situ $\mathrm{KHCO}_{3}$ activation strategy is reported in this paper to prepare $\mathrm{S}$ doped hierarchical porous carbons (S-HPCs) from CTP for SCs application. Unlike the conventional activation method, this method avoids the complicated and harmful acid washing step. The sample obtained at $800^{\circ} \mathrm{C}$ (S$\mathrm{HPC}_{800}$ ) possesses 3D framework structure with hierarchical pores, large specific surface area and $\mathrm{O}, \mathrm{S}$ containing functional groups. Consequently, S-HPC 800 electrode for supercapacitor exhibits high specific capacitance of $246 \mathrm{~F} \mathrm{~g} \mathrm{~g}^{-1}$ at $0.1 \mathrm{~A} \mathrm{~g}^{-1}$ with a capacitance retention of $68.3 \%$ at $40 \mathrm{~A} \mathrm{~g} \mathrm{~g}^{-1}$ and prominent cycle stability with $96.7 \%$ capacitance retention after 10,000 charge-discharge cycles. This work provides an environmentally friendly approach to prepare advanced carbon-based electrode materials from CTP for energy storage devices.

\section{Acknowledgments}

The authors thank the financial supports from the National Natural Science Foundation of China (Nos. 51872005, U1710116 and U1508201).

\section{References}

1. Dong, S.A., He, X.J., Zhang, H.F., et al. (2018) Surface modification of biomass-derived hard carbon by grafting porous carbon nanosheets for highperformance supercapacitors. J. Mater. Chem. A, 6: 15954-15960.

2. Wang, C.J., Liu, F., Chen, J.S., et al. (2020) A graphene-covalent organic framework hybrid for high-performance supercapacitors. Energy Storage Mater., 32: 448-457.

3. Shang, T.X., Xu, Y., Li, P., et al. (2020) A bio-derived sheet-like porous carbon with thin-layer pore walls for ultrahigh-power supercapacitors. Nano Energy, 70: 104531.

4. Shang, Z., An, X.Y., Zhang, H., et al. (2020) Houttuynia-derived nitrogen-doped hierarchically porous carbon for high-performance supercapacitor. Carbon, 161: 62-70.

5. Zhang, P., Zhu, Q.Z., Soomro, R.A., et al. (2020) In situ ice template approach to fabricate 3D flexible MXene film-based electrode for high performance supercapacitors. Adv. Funct. Mater., 2000922.

6. Zhao, J., Jiang, Y.F., Fan, H., et al. (2017) Porous 3D few-layer graphene-like carbon for ultrahigh-power supercapacitors with well-defined structureperformance relationship. Adv. Mater., 29: 1604569.

7. Wang, Y.H., Liu, R.N., Tian, Y.D., et al. (2020) Heteroatoms-doped hierarchical porous carbon derived from chitin for flexible all-solid-state symmetric supercapacitors. Chem. Eng. J., 384: 123263.

8. Liu, B.Q., Zhang, Q., Wang, Z., et al. (2020) Nitrogen and sulfur-codoped porous carbon nanospheres with hierarchical micromesoporous structures and an ultralarge pore volume for high-performance supercapacitors. ACS Appl. Mater. Interfaces, 12: 8225-8232.

9. Huang, Y.B., Pachfule, P., Sun, J.K., et al. (2016) From covalent-organic frameworks to hierarchically porous B-doped carbons: a molten-salt approach. J. Mater. Chem. A, 4: 4273-4279.

10. Cui, C.X., Gao, Y., Li, J., et al. (2020) Origins of boosted charge storage on heteroatom-doped carbons. Angew. Chem. Int. Ed., 59: 7928-7933.

11. Li, J.X., Han, K.H., Wang, D., et al. (2020) Fabrication of high performance structural N-doped hierarchical porous carbon for supercapacitors. Carbon, 164: 42-50.

12. Lu, Y., Liang, J.N., Deng, S.F., et al. (2019) Hypercrosslinked polymers enabled microporedominant N, S co-doped porous carbon for ultrafast electron/ion transport supercapacitors. Nano Energy, 65: 103993.

13. Wei, F., He, X.J., Bi, H.H., et al. (2020) 3D hierarchical carbons composed of cross-linked porous carbon nanosheets for supercapacitors. J. Power Sources, 474: 228698.

14. Wei, F., He, X.J., Ma, L.B., et al. (2020) 3D N, Ocodoped egg-box-like carbons with tuned channels for high areal capacitance supercapacitors. NanoMicro Lett., 12: 82. 
15. Gao, S.S., Tang, Y.K., Wang, L., et al. (2018) Coalbased hierarchical porous carbon synthesized with a soluble salt self-assembly-assisted method for high performance supercapacitors and Li-ion batteries. ACS Sustainable Chem. Eng., 6: 3255-3263.

16. Wang H.R., Yu, S.K., Xu, B. (2016) Hierarchical porous carbon materials prepared using nano- $\mathrm{ZnO}$ as a template and activation agent for ultrahigh power supercapacitors. Chem. Commun., 52: 11512-11515.

17. Zhao, G.Y., Chen, C., Yu, D.F., et al. (2018) One-step production of O-N-S co-doped three-dimensional hierarchical porous carbons for high-performance supercapacitors. Nano Energy, 47: 547-555.

18. Liu, M.Y., Niu, J., Zhang, Z.P., et al. (2018) Potassium compound-assistant synthesis of multi-heteroatom doped ultrathin porous carbon nanosheets for high performance supercapacitors. Nano Energy, 51: 366372.

19. Gopalakrishnan, A., Raju, T.D., Badhulika, S. (2020) Green synthesis of nitrogen, sulfur-co-doped wormlike hierarchical porous carbon derived from ginger for outstanding supercapacitor performance. Carbon, 168: 209-219.

20. Yu, S.K., Sun, N., Hu, L.F., et al. (2018) Self-template and self-activation synthesis of nitrogen-doped hierarchical porous carbon for supercapacitors. J. Power Sources, 405: 132-141.

21. Sheng, L.Z., Jiang L.L., Wei, T., et al. (2017) Spatial charge storage within honeycomb-carbon frameworks for ultrafast supercapacitors with high energy and power densities. Adv. Energy Mater., 7: 1700668.

22. Xie, X.Y., He, X.J., Zhang, H.F., et al. (2018) Interconnected sheet-like porous carbons from coal tar by a confined soft-template strategy for supercapacitors. Chem. Eng. J., 350: 49-56. 